\section{Rehabilitación con coronas completas de metal-cerámica y prótesis parciales removibles convencionales}

Rehabilitation with full of metal-ceramic crowns and conventional removable partial prosthesis

\footnotetext{
Resumen

El tratamiento se realizó en un paciente de sexo femenino de 67 ańos, con buen estado de salud general, portadora de prótesis removible superior incómoda para sus funciones fisiológica y estética. Al examen presenta PPR (prótesis parcial removible) superior de marcado desgaste incisal y oclusal, soporte y retención deficiente, disminución de la DVO (dimensión vertical oclusal), pérdida de contorno facial, edentulismo superior clase II modificación 2 de Kennedy, rebordes conservados, presencia de piezas 1.7, 1.3 y 2.3 ; edentulismo parcial inferior clase III modificación 1 , sin aditamento protésico, rebordes muy reabsorbidos, presencia de piezas $3.8,3.4,3.3,3.2,3.1,4.1,4.2,4.3,4.4,4.5$ y 4.8 .

Se restauró la DVO confeccionando coronas metal cerámica con islas de metal para los apoyos oclusales y brazos recíprocos en los pilares de las piezas 1.7, 3.8 y 4.5. ; y de placas linguales sobre los cíngulos de la piezas 1.3 y 2.3., de la PPR superior e inferior respectivamente. Se construye la morfología oclusal dándole over jet y over bite, desoclusión canina y protección mutua, con cúspides de $30^{\circ}$.

Estéticamente se procedió a restablecer el contorno vestibular superior perdido, con la línea de la sonrisa específica.
}

\footnotetext{
Abstract

The treatment was performed in a female patient 67 years old, with good health status, who came wearing an uncomfortable, for their physiological and aesthetics functions, removable partial dental prosthesis. At initial examination the patients shows an upper removable partial dentures with a great incisal and occlusal wear, poor support and retention, with loss of vertical occlusal dimension, loss of facial contour, Class II Kennedy 2 edentulism, with preserved alveolar ridge and f $1.7 \quad 1.3$ and 2.3 as the only present teeth. In the lower arch There was partial edentulism Class III modification $1 \mathrm{t}$, without actual denture with very reabsorbed alveolar ridge, with $3.8,3.4,3.3,3.2,3.1,4.1,4.2,4.3,4.4,4.5$ and 4.8 as the actual teeth

It was restored VOD by making metal ceramic crowns islands with metal supports and occlusal recipro$\mathrm{cal}$ arms for $1.7,3.8$ and 4.5 abutments. It was made a lingual plate on 1.3 and 2.3 , in the upper and lower removable partial dental prosthesis. We shall now proceed to restore occlusal morphology giving over bite and over jet, cuspid guidance and protective occlusion .

Aesthetically, It was restored upper vestibular contour loss with specific line smile.
}

\section{Lourdes Lara Téllez ${ }^{1}$ Jesús Ochoa Tataje ${ }^{1}$ Jorge Gaitán Velásquez ${ }^{1}$ Marcos Herrera Cisneros ${ }^{1}$ \\ Docentes del Departamento Académico de Estomatología Rehabilitadora, Facultad de Odontología de la UNMSM. Lima, Perú.}

\section{Correspondencia:}

Lourdes Teresa Lara Téllez

Dirección: Calle Las Camelias 174, $2^{\circ}$ piso,

San Isidro, Lima, Perú

Teléfonos: 222-0942/ 422-6739/263-5594

E-mail: loulatel@hotmail.com

Palabras clave: Prótesis parcial removible (PPR), conector mayor combinado (placa palatina anterior y barra palatina posterior), dimensión vertical oclusal (DVO), dimensión vertical postural (DVP), espacio libre (EL).

Keywords: Removible Partial prosthesis (RPP), major combined conector, vertical oclusal dimension (VOD), vertical postural dimension (DPV), free space (FE).

\section{Introducción}

El examen clínico secuencial, riguroso y detallado, complementado con los exámenes auxiliares requeridos, es imprescindible para lograr que no se pierda ningún detalle que en su momento va ser determinante para la planificación del tratamiento. ${ }^{1-3}$

El requerimiento de rehabilitación protésica hace que el profesional obtenga alternativas de tratamiento, para que el paciente pueda escoger el adecuado según su necesidad, y economía. ${ }^{4-7}$

La rehabilitación protésica con coronas individuales de metal cerámica combinada con PPR superior e inferior con base metálica, provee al paciente la solución a su problema bucal, de manera eficiente tanto funcional como estética. ${ }^{3,5,6,8,9}$

La disminución de la DVO es común en los pacientes que han sufrido pérdida de piezas dentales, originando migraciones y desgastes contribuyendo a la maloclusión, adicionado con prótesis de larga data, desadaptadas, la mayoría sin antagonista, haciendo más complejo el problema. ${ }^{4,6,10-12}$

El éxito será el resultado de la conjunción de lo que el profesional ofrece al paciente, y la motivación lograda en el paciente para la conservación de sus prótesis y tejidos remanentes a través del tiempo. ${ }^{1,6,12,13-16}$

Objetivos del tratamiento

1. Planificar multidisciplinariamente el tratamiento integral del paciente.
2. Mejorar la salud bucal del paciente mediante tratamiento periodontal.

3. Realizar el tratamiento operatorio y endodóncico.

4. Colocar restauraciones fijas individuales en las piezas con inclinaciones moderadas para la recepción de las prótesis parciales removibles.

5. Devolver la dimensión vertical oclusal mediante restauraciones individuales de metal cerámica, y placas linguales integradas a las prótesis parciales removibles.

6. Devolver la fisiología bucal.

7. Devolver la estética de la zona vestibular anterior. 
8. Motivar y educar al paciente para la conservación correcta de sus prótesis y tejidos remanentes, al igual que para que asista puntualmente a sus controles periódicos.

\section{Caso clínico}

Paciente de sexo femenino de 67 años de edad, con antecedente de extracciones dentales por caries y enfermedad periodontal. Acude a la consulta por incomodidad al masticar e inconformidad con la estética, porta PPR superior en malas condiciones, sin prótesis inferior (Figs 1, 2A y B).

$\mathrm{Al}$ examen se observa presencia de piezas $1.7,1.3$ y 2.3 en la arcada superior (Fig. 3); y las piezas 3.8, 3.4, 3.3, 3.2, $3.1,4.1,4.2,4.3,4.4,4.5$ y 4.8 , en la arcada inferior (Fig. 4). Pieza 1.7 mesializada y vestibularizada, con faceta de desgaste en la vertiente distal de la cúspide mesiopalatina, en 3.8 y 4.8 mesio-lingualizadas moderadamente, pieza 4.5 severamente inclinada hacia lingual. Presencia de lesiones no cariosas en zona cérvico vestibular de 3.4, y 4.4; y 4.5, presencia de cavidad cariosa de 2 do grado en oclusal de 1.7.

Al examen de la oclusión se observa contacto en retrusiva entre piezas $1.7 \mathrm{y}$ 3.8 produciendo deslizamiento hacia el lado izquierdo hasta llegar a la máxima intercuspidación.

$\mathrm{Al}$ movimiento de lateralidad izquierda se observa interferencia en lado de balance a nivel de pieza 1.7 vs. pieza 4.8. A la palpación muscular refiere dolor en el pterigoideo externo derecho. No presenta ruidos articulares.

Se observa pérdida de la DVO, mostrando pseudoprotrusión mandibular (bis a bis), retenedor antiestético en canino sup., abfracción de dientes inferiores. (Fig. 1). Deformidad de la línea de la sonrisa, plano oclusal distorsionado, ausencia de prótesis inferior, (Figs. 2 A y B) lingualización de molares inf., apiñamiento de dientes antero inferior, frenillo lingual de inserción alta, produciendo dificultad para el diseño del conector mayor inferior (Fig. 4).

Con respecto al registro de la dimensión vertical (DVP-DVO), se encontró que el espacio libre (EL) era muy amplio (entre 7 y 8 $\mathrm{mm}$,) demostrando la pérdida de DVO.

\section{Diagnóstico}

Del estado general de salud:

Paciente de sexo femenino de 67 años de edad, con aparente buen estado de salud general, lúcida y orientada en el tiempo, espacio y persona; sin riesgo al tratamiento estomatológico.

Del estado de salud odontoestomatológico: Paciente con:

Dientes con abfracción, caries dental y malposición.

Pieza 4.5 severamente inclinada hacia lingual.

Gingivitis generalizada.

Oclusión alterada.

Edéntula parcial superior clase II modificación 2 de Kennedy e inferior clase III modificación 1 de Kennedy.

Frenillo lingual de inserción alta.

Rebordes residuales inferiores reabsorbidos y superiores conservados.

\section{Plan de tratamiento}

Paciente sin riesgo al tratamiento estomatológico, plan de tratamiento:

1. Fisioterapia oral, enseñanza de técnica de cepillado y uso del hilo dental. Enseñanza higiene de la prótesis inicial como modelo.

2. Profilaxia oral.

3. Operatoria dental con resina compuesta de la pieza 1.7 por caries dental, ya que sería posteriormente tallada para corona; y de las piezas $3.4,4.4$ y 4.5 por abfracción.

4. Endodoncia por necesidad protésica de la pieza 4.5.

5. Espigo muñón prefabricado metálico en la pieza 4.5 .

6. Eliminación de interferencia oclusal de la pieza 1.7 y 4.8 .

7. Confección de coronas provisionales acrílicas en las piezas dentales talladas y rebase con acrílico de la prótesis parcial removible inicial para uso provisional, según diseño protésico.

8. Confección de coronas de metal porcelana con islas de metal para conectores y apoyos en las piezas $1.7,3.8,4.5$ y 4.8 , cambiando la dirección axial de la corona según diseño protésico preliminar.

9. Confección de prótesis parcial removible superior e inferior.

10. Instalación de los aditamentos protésicos.

11. Reforzamiento de la enseñanza de la técnica de cepillado y uso de hilo dental, además del aseo de las prótesis.
12. Control semanal.

13. Control mensual.

14. Control trimestral.

15. Control semestral.

\section{Procedimientos clínicos}

Se inicia el tratamiento consentido con la fase 1 y 2 del plan establecido, educación para el aseo de la prótesis que venía portando, profilaxis para combatir la gingivitis moderada que presentaba.

Siguiente cita: toma modelos de estudio y registros, para el montaje en ASA (Fig. 5). Usando placas base y rodetes de oclusión y contorno superior e inferior, se registró en relación céntrica, para aumentar la DVO disminuida, elaborando un nuevo esquema oclusal.

Operatoria en 1.7 oclusal con resina compuesta, endodoncia en pieza 4.5 debido a la posible exposición pulpar durante la preparación biostática, es decir, durante la elaboración de los planos guía. Preparación, cementado del espigo metálico prefabricado, con el muñón de resina orientado según el diseño; gracias a la inclinación natural de la corona de las piezas inferiores hacia lingual, permitiendo este cambio de angulación coronaria sin producir esfuerzos perjudiciales. Seguidamente se realizó la eliminación de las interferencias y contactos prematuros.

Paralelizado, diseño definitivo, se determina la cantidad a aumentar de la DVO, confección de provisionales (Fig. 6), para proceder a la preparación de las piezas para restauraciones completas de metal porcelana, tomando en cuenta que los dientes involucrados, tendrían una nueva posición axial coronaria dada su mala posición en el arco.

La cantidad en milímetros a aumentar de la dimensión vertical oclusal se determinó mediante la simple fórmula: $\mathrm{DVO}+\mathrm{EL}=\mathrm{DVP}$.

Donde DVO es la dimensión vertical oclusal; EL es el espacio libre; y DVP es la dimensión vertical postural.

Siendo en la paciente la DVP de $79 \mathrm{~mm}$ y el EL debiera ser entre 2 y $4 \mathrm{~mm}$, la DVO debiera fluctuar entre 75 Y $77 \mathrm{~mm}$, pero la paciente tiene $73 \mathrm{~mm}$, entonces existe una disminución de DVO entre 2 a $4 \mathrm{~mm}$.

\section{Diseño}

Previo paralelizado, se procedió al análisis de los modelos de estudio, y se con- 
cluyó hacer el diseño de la ARCADA SUPERIOR (Fig. 9).

- $\quad$ Eje de inserción: único.

- Conector mayor combinado: una placa palatina anterior y una barra palatina posterior.

- Retenedores directos: tipo barra en I en los caninos, con placa palatina para aumento de la dimensión vertical y placa proximal mesial y distal a cada pieza. Retenedor tipo Acker en la pieza 1.7 sobre una corona metal cerámica elaborada para la recepción de la PPR.

- Y en la ARCADA INFERIOR: (Fig. 10).

- Eje de inserción: único.

- Conector mayor: placa lingual, debido a la posición alta del frenillo lingual, debiéndose remodelar las piezas anteriores inferiores para eliminar las interferencias durante el asentamiento de la PPR. (Fig. 7).

- Retenedores directos: en las piezas 3.8 Acker sobre la corona metal cerámica preparada; pieza 3.4 tipo barra en I, debido a la brecha edéntula larga, para que funcionara como rompe fuerzas; pieza 4.5 Acker sobre corona metal cerámica; y pieza 4.8 Acker modificado, con dos apoyos oclusales en mesial y distal, para adicionar la altura dental.

Preparación biostática y confección de provisionales

La preparación de los dientes se realizó tomando en cuenta el eje de inserción de la futura PPR tanto superior como inferior.

Confección de coronas provisionales acrílicas de curado rápido, adecuándose tanto al eje de inserción y alturas según encerado diagnóstico previo, como al retenedor de la PPR inicial rebasada que actuó como prótesis provisional.

\section{Impresiones}

Las impresiones definitivas se realizan con silicona de condensación, técnica del hilo retractor para la elaboración de las coronas de metal porcelana; se procedió al montaje en ASA y se remitió al laboratorio con las respectivas indicaciones.

Una vez probadas y "ajustadas" las coronas de metal cerámica, se colocaron en los pilares y se procedió a la toma de impresión de arrastre con silicona de condensación y cubetas individuales (Fig. 8 A y B), previa preparación biostática de la boca, es decir preparaciones de los lechos en las piezas caninos superiores, premolares inferiores y remodelado de la zona antero inferior para facilitar la adaptación de la placa lingual, diseño que, por tener el frenillo lingual de inserción alta, fue el más adecuado (Fig. 7).

Prueba de la estructura metálica

Prueba de la estructura metálica superior e inferior, con las coronas asentadas. Se seleccionó el color de dientes y se adaptó en las bases metálicas, los rodetes de cera para la toma final de registro y articulado en ASA; se debió considerar muy exactamente el contorno vestibular para dar la estética deseada, incluyendo la sobremordida horizontal y vertical.

La siguiente cita consistió en la prueba de dientes en cera, ajuste oclusal y estético, una vez aprobado también por el paciente, se procedió a ordenar el acrilizado.

Instalación de las prótesis

En la cita de instalación se consideró:

Asentamiento exacto de las coronas y PPR armonizando exactamente cada una de ellas, exactitud del color, armonía del contorno vestibular, ajuste oclusal, tanto en posición céntrica como en movimientos excéntricos, estética adecuada.(Figs. 9 y 10).

Cementado de coronas metal cerámicas, instalación de las PPR superior e inferior. (Figs.11 y 12).

Se dio al paciente instrucciones por escrito acerca de la conservación y aseo de las prótesis y de sus tejidos remanentes. Se citó a la semana, luego al mes, a los tres meses, y finalmente a cada seis meses.

\section{Resultados}

En la rehabilitación de casos clínicos como el presentado, la prótesis combinada se convierte en una solución adecuada por su ejecución relativamente sencilla con resultados duraderos en el tiempo, además de no ser un tratamiento riesgoso para la salud ni oneroso económicamente.

En el aspecto de fisiología mandibular se cumple con todos los parámetros necesarios para establecer y mantener una adecuada función del sistema estomatognático.

En el sentido estético, también cumplimos consiguiendo uniformidad de características entre los dientes artificiales fijos y removibles con los naturales; además los materiales odontológicos posibilitan una transición de estructuras sin que se perciba con facilidad el cambio.
El trabajo también tiene efectos extra-orales cuando la paciente atenúa las líneas de expresión mostrando una facies mejorada.

\section{Discusión}

Las prótesis combinadas: fija y removible resulta ser una alternativa valedera en aquellos pacientes que no puedan por diversos motivos, acceder a prótesis sofisticadas o tratamientos con implantes. ${ }^{2,6-9}$

Tratamientos de conductos en dientes muy inclinados puede ser indicada siempre y cuando el tratamiento protésico lo justifique ampliamente, evitando así una brecha amplia y/o extremos libres, que dificulten la retención, soporte y estabilidad. ${ }^{1,2,7-11}$

El diseño de retenedores a barra del tipo I en dientes anteriores, no se aparta de la estética, más aún en pacientes con labios largos, pues habiendo brechas edéntulas amplias, sería imposible otro tipo de retenedor directo extracoronario que nivele su eficiencia funcional. ${ }^{2-5,8,9,13,15}$

El aumento de la DV se logra con coronas coladas, apoyos oclusales y placas linguales colocadas armoniosamente para lograr excelente oclusión acorde con la fisiología del paciente. ${ }^{12,14,16}$

\section{Conclusiones}

Los resultados nos permiten hacer las siguientes conclusiones:

- La prótesis parcial removible es una alternativa para el tratamiento del edéntulo parcial.

- La atención a los detalles en todas las etapas del tratamiento (puede) asegura una rehabilitación protésica exitosa.

- Es ventajoso combinar restauraciones individuales de cobertura total tipo metal cerámica, con prótesis parcial removible, cuando existen migraciones y malposiciones moderadas de los pilares.

- Se logra restaurar la DVO disminuida con prótesis combinadas fija y removible.

- Se consigue el funcionamiento armonioso y fisiológico de las prótesis combinadas.

- Es necesario educar al paciente sobre la importancia de un buen mantenimiento de los elementos protésicos y de las estructuras bucales residuales.

- Se puede atenuar las líneas de expresión faciales con rehabilitaciones ora- 
les adecuadas, sin necesidad de recurrir a tratamientos plásticos.

\section{Referencias bibliográficas}

1. Henderson, D. "Prótesis Parcial Removible según Mc Cracken" Edit. Mundi S.A.I.C. y F. 1985

2. Loza, D. Valverde R. "Diseño de prótesis parcial removible" Edit. $\mathrm{Ri}$ pano, 2007.

3. Mallat Desplats, E. "Prótesis parcial removible", Edit. Harcourt Brace de España. $1^{\circ}$ impresión 1998.

4. García Michelsen JL. "Diseño de Prótesis Parcial Removible" Edit. Amolca, 2005

5. Kratochvil, J. Prótesis parcial removible. 1 ra ed. editorial Interamericana; 1989.

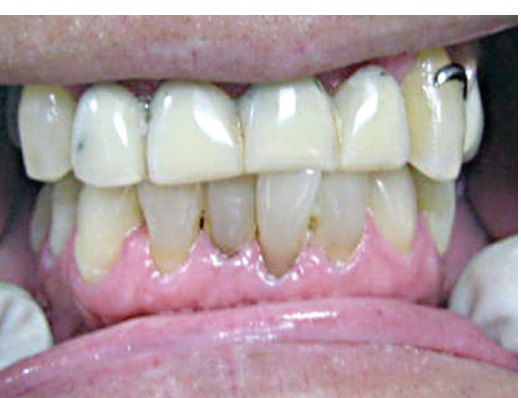

Fig. 1. Vista frontal inicial con PPR en uso.



Fig. 3. Vista oclusal superior nótese la vestibularización de la pieza 1.7 y la profundidad de la bóveda palatina.

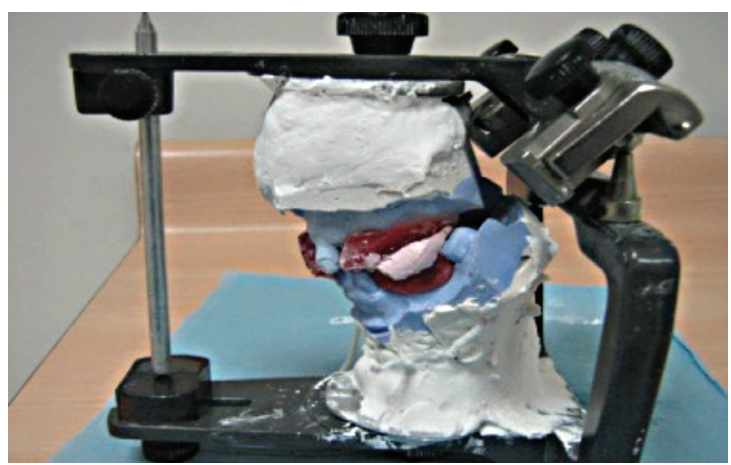

Fig. 5. Montaje de modelos de estudio en ASA.
6. Clínicas Odontológicas de Norteamérica, "Reconstrucción Bucal completa: Fija y removible" Mc Graw-Hill. 1987.

7. Pegoraro LF. "Prótesis Fija" Edit. Artes Médicas Latinoamérica, $1^{\circ}$ edición, Sao Paulo 2001.

8. Loza Fernández, D. "Prótesis parcial removible". Caracas: Editorial Actualidades Médico Odontológicas Latinoamérica; 1992.

9. Rendón Yudice, R. "Prótesis parcial removible: conceptos actuales, atlas de diseño" Editorial Panamericana, 2007

10. Mezzomo E. "Rehabilitación oral para el clínico" Edit. Amolca 2003.

11. Koth D L. Malone W. F. P., “TYLMANS Teoría y práctica en prostodoncia fija" $8^{\circ}$ ed. Edit. Actualidades Médico Odontológicas Latinoamérica, C. A., 1994

\section{Secuencia del caso clínico}

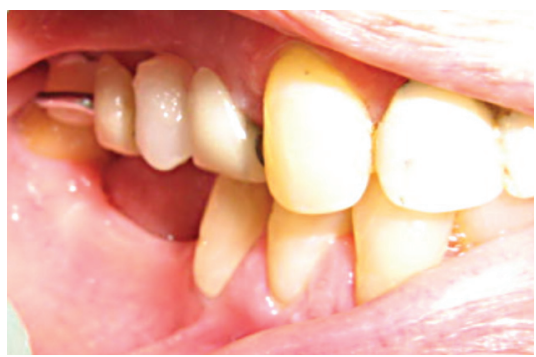

Fig. 2A
12. Cadalfach GE. "Manual clínico de prótesis Fija” España: Edit. Harcourt Brace 1997

13. Graber G. Atlas de prótesis parcial. 2a ed. Salvat Editores; 1993.

14. Miller, E. Prótesis parcial removible. México, D.F: Editorial Interamericana; 1975.

15. Kaiser, F. PPR en el laboratorio. $2^{\mathrm{a}} \mathrm{ed}$ Curitiba: Editora Maio; 2002.

16. Ivoclar Vivadent AG en colaboración con el Prof. Dr. Pfister (Área de trabajo microbiología oral), Prof. Dr. Welter (Área de trabajo materiales dentales) y Dra. Hinz (Área de trabajo prótesis clínica) de la Universidad Jena/Alemania. "Una guía práctica para cuidar su prótesis" 2005

Fecha de recepción: 23 de junio 2009

Fecha de aprobación: 5 de enero 2010

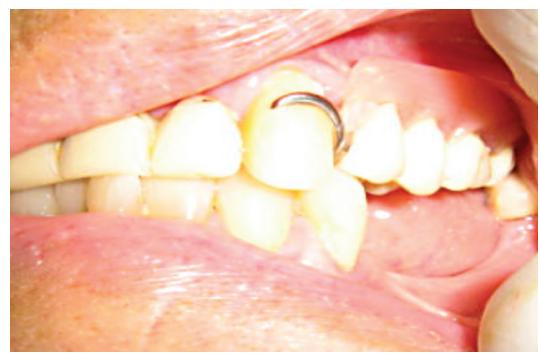

Fig. 2B

Fig. 2. A y B. Vista lateral derecha e izquierda con la PPR superior inicial desadaptada.

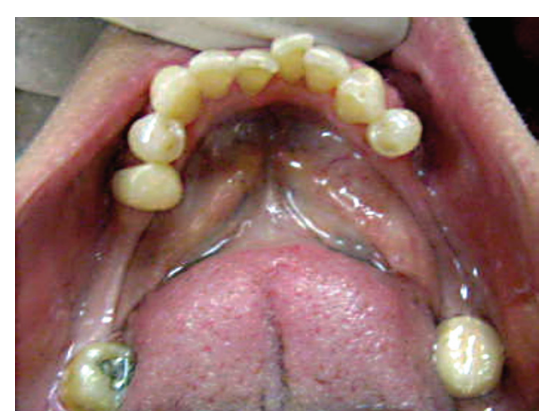

Fig. 4. Vista oclusal inferior, con piezas 3.8, 4.5 y 4.8 lingualizadas y mesializadas, nótese también la inserción alta del frenillo lingual y el apiñamiento de los incisivos anterior.

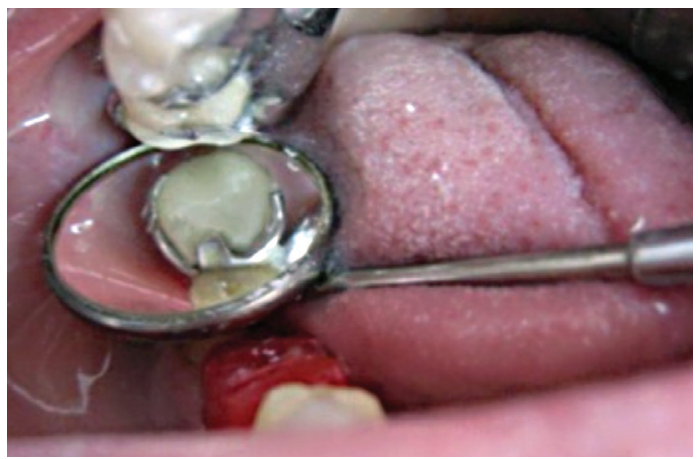

Fig. 6. Provisional de acrílico adaptado a la PPR provisional. 

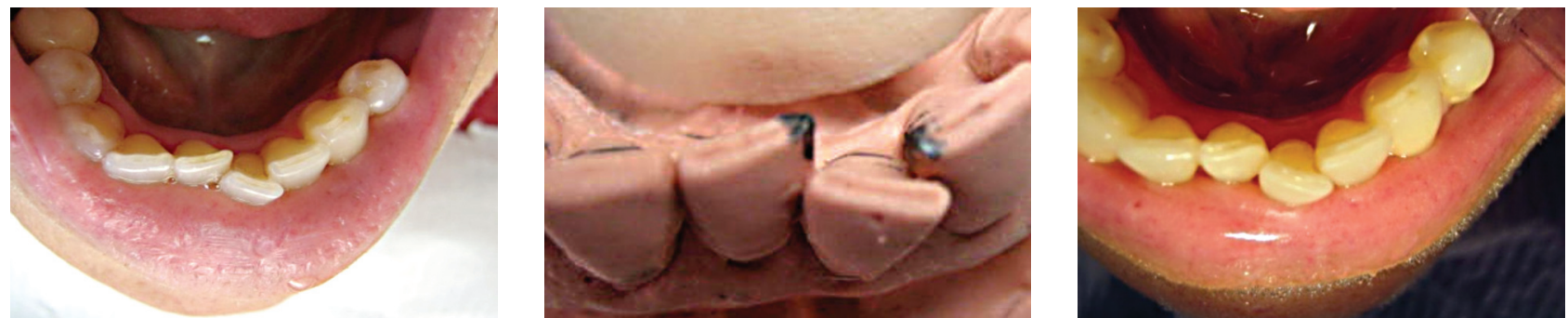

Fig. 7. Debido al apiñamiento del sector antero inferior, fue necesario el remodelado de las piezas dentales, como parte de la preparación biomecánica.

A

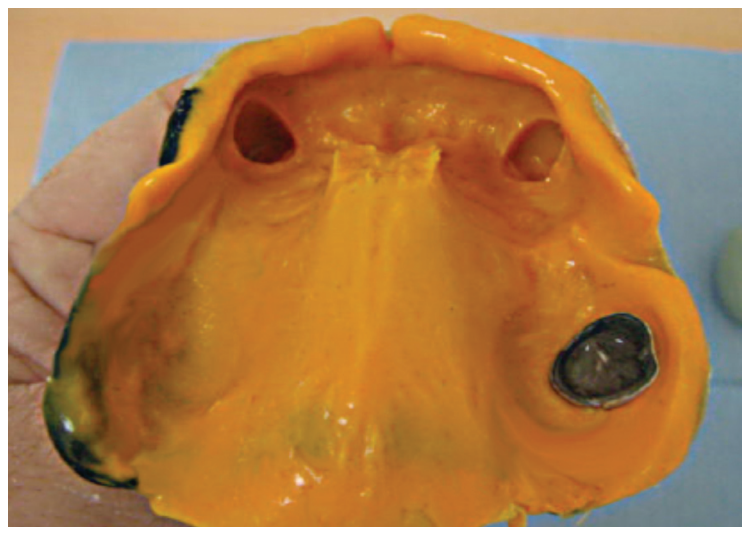

B

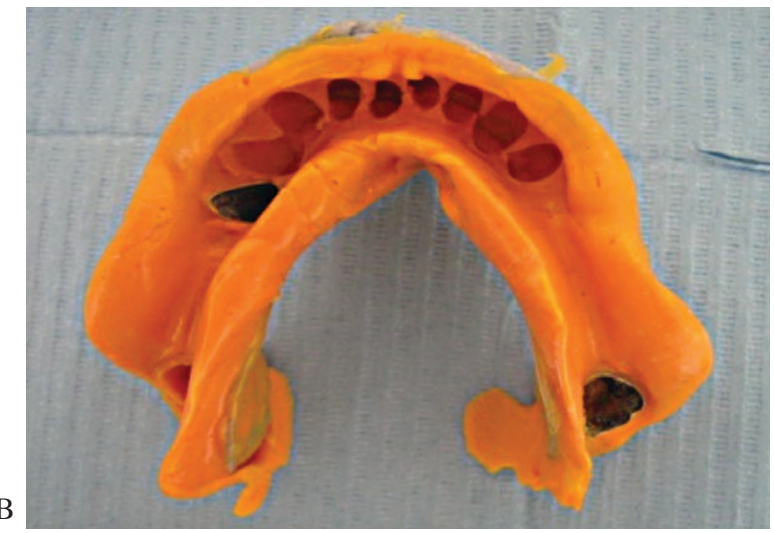

Fig. 8. Impresión de arrastre para la confección de las estructuras metálicas de las PPR A. superior y B. Inferior.

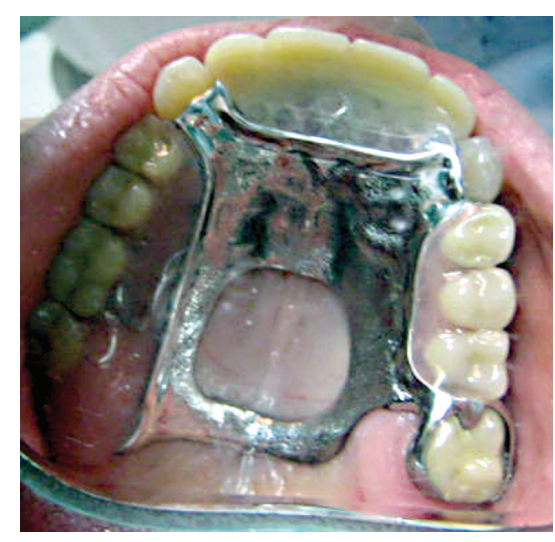

Fig. 9. Vista oclusal de la arcada superior con prótesis instaladas.

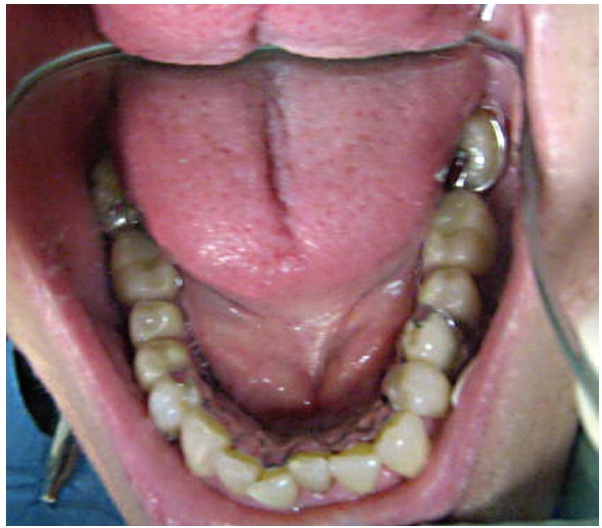

Fig. 10. Vista oclusal de la arcada inferior.
A

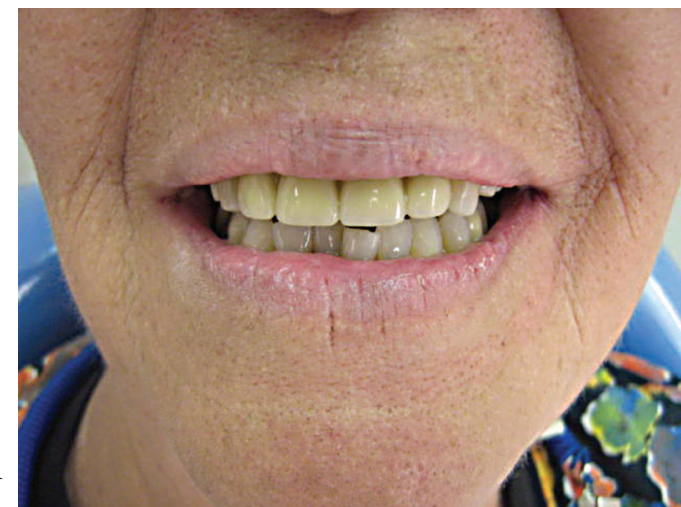

$\mathrm{B}$

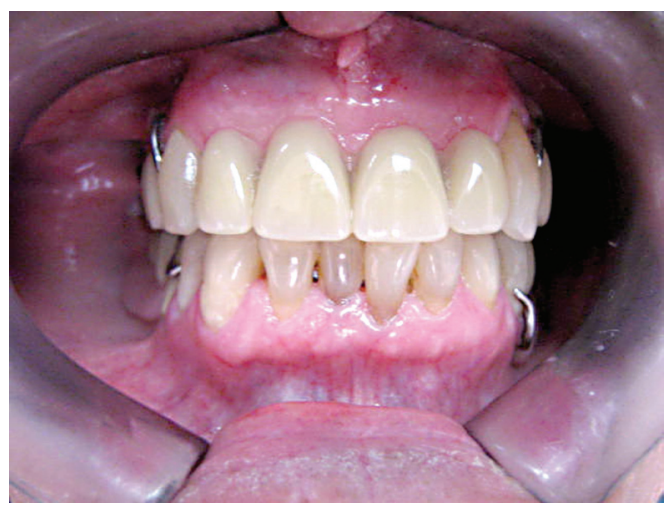

Fig. 11-12. Caso terminado, obsérvese la recuperación de la dimensión vertical A. Postural, B. Oclusal; y la armonía en la estética. 